\title{
Barriers and facilitators to implementation of epilepsy self-management programs: a systematic review using qualitative evidence synthesis methods
}

\author{
Allison A. Lewinski 1*, Abigail Shapiro ${ }^{1,2}$, Jennifer M. Gierisch ${ }^{1,3,4}$, Karen M. Goldstein ${ }^{1,4}$, Dan V. Blalock ${ }^{1,5}$, \\ Matthew W. Luedke ${ }^{6,7}$, Adelaide M. Gordon' ${ }^{1}$, Hayden B. Bosworth ${ }^{1,3,5,8,9}$, Connor Drake ${ }^{9,10}$, Jeffrey D. Lewis ${ }^{11}$, \\ Saurabh R. Sinha, ${ }^{6,7}$, Aatif M. Husain ${ }^{6,7,12}$, Tung T. Tran ${ }^{6,7}$, Megan G. Van Noord ${ }^{13}$ and John W. Williams Jr J,4,5
}

\begin{abstract}
Background: Epilepsy affects nearly 50 million people worldwide. Self-management is critical for individuals with epilepsy in order to maintain optimal physical, cognitive, and emotional health. Implementing and adopting a selfmanagement program requires considering many factors at the person, program, and systems levels. We conducted a systematic review of qualitative and mixed-methods studies to identify facilitators and barriers that impact implementation and adoption of self-management programs for adults with epilepsy.

Methods: We used established systematic review methodologies for qualitative and mixed-methods studies. We included studies addressing facilitators (i.e., factors that aided) or barriers (i.e., factors that impeded) to implementation and adoption of self-management interventions for adults with epilepsy. We conducted a narrative thematic synthesis to identify facilitators and barriers.

Results: The literature search identified 2700 citations; 13 studies met eligibility criteria. Our synthesis identified five themes that categorize facilitators and barriers to successful implementation epilepsy self-management: (1) relevance, intervention content that facilitates acquisition of self-management skills; (2) personalization, intervention components that account for the individual's social, physical, and environmental characteristics; (3) intervention components, components and dosing of the intervention; (4) technology considerations, considerations that account for individual's use, familiarity with, and ownership of technology; and (5) clinician interventionist, role and preparation of the individual who leads intervention. We identified facilitators in 11 of the 13 studies and barriers in 11 of the 13 studies and classified these by social-ecological level (i.e., patient/caregiver, program, site/system).

(Continued on next page)
\end{abstract}

\footnotetext{
* Correspondence: allison.lewinski@duke.edu

${ }^{1}$ Durham Center of Innovation to Accelerate Discovery and Practice

Transformation, Durham Veterans Affairs Medical Center, Durham, NC, USA

Full list of author information is available at the end of the article
}

(c) The Author(s). 2020 Open Access This article is licensed under a Creative Commons Attribution 4.0 International License, which permits use, sharing, adaptation, distribution and reproduction in any medium or format, as long as you give appropriate credit to the original author(s) and the source, provide a link to the Creative Commons licence, and indicate if changes were made. The images or other third party material in this article are included in the article's Creative Commons licence, unless indicated otherwise in a credit line to the material. If material is not included in the article's Creative Commons licence and your intended use is not permitted by statutory regulation or exceeds the permitted use, you will need to obtain permission directly from the copyright holder. To view a copy of this licence, visit http://creativecommons.org/licenses/by/4.0/. The Creative Commons Public Domain Dedication waiver (http://creativecommons.org/publicdomain/zero/1.0/) applies to the data made available in this article, unless otherwise stated in a credit line to the data. 
(Continued from previous page)

Conclusion: Identification of facilitators and barriers at multiple levels provides insight into disease-specific factors that influence implementation and adoption of self-management programs for individuals with epilepsy. Our findings indicate that involving individuals with epilepsy and their caregivers in intervention development, and then tailoring intervention content during the intervention, can help ensure the content is relevant to intervention participants. Our findings also indicate the role of the clinician (i.e., the individual who provides self-management education) is important to intervention implementation, and key issues with clinicians were identified as barriers and opportunities for improvement. Overall, our findings have practical value for those seeking to implement and adopt self-management interventions for epilepsy and other chronic illnesses.

Systematic review registration: PROSPERO registration number is CRD42018098604.

Keywords: Epilepsy, Self-management, Qualitative research

\section{Contributions to the literature}

- Patient, caregiver, and clinician involvement may improve implementation and adoption of epilepsy self-management interventions

- A flexible self-management approach with tailoring to individual patients may address concerns regarding cognitive limitations, use of technology, and relevance of self-management information

- Clinicians involved in self-management interventions need appropriate training, dedicated clinical time, and to be provided with clearly written standardized protocols that articulate the clinician's role in the intervention

- Technology use for self-management is highly dependent on individual characteristics such as familiarity and ownership of technological devices (e.g., mobile phones, computers)

\section{Background}

The World Health Organization estimates that epilepsy affects about 50 million people worldwide [1]. Individuals with epilepsy have poorer health outcomes and report a poorer quality of life [2-4]. Self-management is important for individuals with epilepsy in order to maintain optimal physical, cognitive, and emotional health following diagnosis [5, 6]. Engagement in self-management may increase self-efficacy, medication adherence, avoidance of seizure triggers, and improves patient and family knowledge about when to seek urgent medical care $[7,8]$. For instance, education based-approaches positively impact selfmanagement, whereas psychosocial therapy-based approach positively impact quality of life $[9,10]$. A recent Cochrane review identified weak support for intervention strategies such as using epilepsy-trained nurses and educational interventions to support self-management in adults with epilepsy [11]. Yet, rates of self-management and adherence to treatment regimens remain suboptimal among individuals with epilepsy, leading to increased medical care and premature death $[5,6]$.

As with many chronic health conditions, challenges to self-management for adults with epilepsy include low health literacy, poor social support, low levels of education, medication and disease-related side effects, and low socioeconomic status [12-18]. Patients with epilepsy have additional disease-specific self-management concerns which can impact engagement in, and adherence to, selfmanagement [19]. The effectiveness of self-management in adults with epilepsy may be influenced by commonly co-occurring medical conditions such as traumatic brain injury, mood disorders, associated cognitive impairment, and/or impulse-control issues. Further complicating selfmanagement plans, high levels of disease-related stigma, driving or mobility restrictions, and social isolation may serve as barriers to attending intensive epilepsy selfmanagement programs and/or medical appointments that occur in-person and/or require a sustained time commitment [20-22]. Thus, engagement in self-management and treatment adherence may be positively influenced when self-management programs are informed by knowledge of epilepsy-specific considerations and how these considerations may influence self-management.

A possible solution to epilepsy-specific self-management concerns may be self-management programs offered within the context of clinicians providing health care for patients with epilepsy. Implementing self-management programs in health care systems requires consideration of many multi-level factors [23]. Facilitators and barriers to implementation can occur at any level of an intervention, including the person (patient or caregiver), program, and system levels. Research has shown the importance of allocating resources, balancing context-specific adaptation and program fidelity, and involving key stakeholders during initial and sustained implementation and adoption [24-27]. Although scientific organizations recommend self-management support [28-30], there is little guidance about how to implement these programs, and we identified no prior systematic reviews that focused on this issue. 
We conducted a systematic review of quantitative, qualitative, and mixed-methods studies to examine the barriers and facilitators to implementing and adopting epilepsy self-management programs in large health care systems. We chose qualitative evidence synthesis methods to incorporate evidence from multiple study designs that addressed the same overarching concept of implementation and adoption of epilepsy selfmanagement programs. Our study addresses gaps in understanding facilitators and barriers at the person, program, and system levels that impact implementation and adoption of epilepsy self-management programs.

\section{Methods}

This systematic review was part of a Veterans Health Administration (VHA)-funded report available online (www.hsrd.research.va.gov/publications/esp) [9, 10]. The purpose of the systematic review was to address gaps in evidence regarding the efficacy and effectiveness of selfmanagement programs for adults with epilepsy, synthesize the current evidence on self-management programs for patients with epilepsy, and identify potential barriers in the adoption of these programs within a health care system. We developed and followed a standard protocol for this review in collaboration with stakeholders (PROSPERO: CRD42018098604). We adhered to the Preferred Reporting Items for Systematic Reviews and Meta Analyses (PRISMA) guidelines [31] (Additional file 1).

\section{Literature search and study selection}

The conceptual model, literature search, and study selection methods for the systematic review are described in greater detail in the full monograph $[9,10]$. A full list of inclusion and exclusion criteria (i.e., population, intervention, comparator, outcome, timing, and setting) is listed in Table 1. The major criteria for eligibility included randomized or quasi-experimental studies that enrolled adults with epilepsy and evaluated selfmanagement interventions and reported a relevant clinical, process, or economic outcome. Eligible interventions were required to have a primary focus on selfmanagement of epilepsy, could not be education-only or general medical care interventions, and could not focus primarily on a comorbid psychological diagnosis. Additionally, eligible studies focused on adults (aged $\geq 18$ ) with new or chronic epilepsy, family members and/or caregivers of adults with epilepsy, and/or stakeholders involved in implementation of epilepsy self-management programs. For this specific analysis, we included additional observational designs and qualitative studies that addressed facilitators and barriers to intervention implementation and adoption. We operationalized selfmanagement as programs that facilitated the provision of epilepsy-specific knowledge and skills that assisted an individual and/or caregiver in incorporating epilepsy self-management behaviors into daily life. Our definition was based upon a conceptualization of self-management by Jonkman and colleagues [32]; however, we modified the definition to only include one component beyond knowledge acquisition $[9,10]$. We operationalized implementation and adoption as the uptake, and continued use, of an evidence-based intervention in health care practice [34].

For this systematic review, we collaborated with a medical librarian to develop a search strategy for each database (Additional file 2) [9, 10]. We searched MEDLINE $^{\oplus}$ (via PubMed ${ }^{\circ}$ ), PsycINFO, Cochrane Central Register of Controlled Trials (CENTRAL), and CINAHL in April 2018, and the MEDLINE search was updated in March 2019. Citations and the full-text of potentially eligible studies were evaluated by two investigators. We resolved disagreements using consensus between two investigators, and involved a third investigator as needed. All articles that met eligibility requirements and addressed facilitators and barriers to implementation and adoption were included for data abstraction.

\section{Data extraction strategy}

Data abstraction was completed by a team of two coinvestigators (AAL, AS) who had experience in qualitative methodology, under the guidance of the primary investigator (JWW). Data from published reports were entered into a Microsoft Excel spreadsheet created for this project. Abstracted data included study purpose, study design and details, participant eligibility criteria, recruitment strategy, control group, participant type (i.e., patient, caregiver, clinician, researcher), sample size and demographics, main findings salient to facilitators and barriers of implementation and adoption, and other comments. We abstracted implementation and adoption barriers (i.e., description of themes or factors that impeded the use and implementation of the intervention as reported in the study's results and/or findings sections) and facilitators (i.e., description of themes or factors that aided the use of the intervention as reported in the study's results and/or findings sections) to the implementation of self-management interventions (as distinct from barriers and facilitators of an individual engaging in self-management behaviors). Data were entered independently by one investigator, and then independently reviewed by a second investigator. Following the completion of data entry, the two investigators met to discuss abstracted data; disagreements were resolved by consensus.

\section{Risk of bias}

To evaluate the risk of bias (ROB) across included studies, we employed ROB instruments appropriate for each 
Table 1 Eligibility criteria (reproduced with permission from Luedke et al. [9])

\begin{tabular}{|c|c|c|}
\hline $\begin{array}{l}\text { Study } \\
\text { Characteristic }\end{array}$ & Include & Exclude \\
\hline Population & $\begin{array}{l}\text { - Adults (aged } \geq 18 \text { ) with new or chronic epilepsy } \\
\text { - Family members and/or caregivers of those with epilepsy } \\
\text { - Stakeholders involved in implementation (e.g., neurologists, health } \\
\text { coaches, nurses, administrators) }\end{array}$ & $\begin{array}{l}\text { - Children } \\
\text { - Populations with < 70\% adults } \\
\text { - Severe learning disabilities } \\
\text { - Non-epileptic seizures (i.e., psychogenic seizures) } \\
\text { - Populations who have been recruited for depression or who } \\
\text { have major mental illness (e.g., bipolar, major depressive } \\
\text { disorder, schizophrenia) }\end{array}$ \\
\hline Intervention & $\begin{array}{l}\text { Self-management defined as interventions that aim to equip } \\
\text { patients with skills to actively participate and take responsibility in } \\
\text { the management of epilepsy in order to function optimally } \\
\text { through at least knowledge acquisition and a combination of } 1 \text { or } \\
\text { more of the following: } \\
\text { - Stimulation of independent sign/symptom monitoring } \\
\text { - Medication management } \\
\text { - Enhancing problem-solving and decision-making skills for epilepsy } \\
\text { treatment management, safety promotion (e.g., driving) } \\
\text { - Changing health behaviors (including stress management, sleep, } \\
\text { substance use) } \\
\text { Examples include: } \\
\text { - Psychoeducation (e.g., cognitive behavioral therapy) } \\
\text { - Behavioral interventions (e.g., adherence strategy training) } \\
\text { - Personalized care plan development and coaching }\end{array}$ & $\begin{array}{l}\text { - Multicomponent interventions that include self-management } \\
\text { but where self-management is not the primary intervention } \\
\text { - Cognitive behavioral therapy focused on comorbid mental } \\
\text { illness in patients with epilepsy (e.g., depression in patients with } \\
\text { epilepsy) } \\
\text { - Education-only interventions } \\
\text { - General care delivery interventions (e.g., introducing specialist } \\
\text { nurse practitioner or implementation of clinical practice } \\
\text { guidelines) }\end{array}$ \\
\hline Comparator & Any (usual care, attention control, active intervention) & None \\
\hline Outcomes & $\begin{array}{l}\text { Any relevant clinical, process, or economic outcome to epilepsy } \\
\text { self-management interventions }\end{array}$ & None \\
\hline Timing & Any & Any \\
\hline Setting & $\begin{array}{l}\text { - Delivered in person (individual or group) in outpatient settings, or } \\
\text { remotely via telehealth technology (e.g., mobile or internet) } \\
\text { - Delivered by health care team members or trained lay workers }\end{array}$ & $\begin{array}{l}\text { - Inpatient } \\
\text { - Delivered only in emergency departments }\end{array}$ \\
\hline Design $^{b}$ & $\begin{array}{l}\text { - Randomized trials } \\
\text { - Nonrandomized trials } \\
\text { - Controlled before-after studies } \\
\text { - Prospective cohort study if it includes a properly adjusted analysis } \\
\text { - Qualitative and survey designs if specifically addressing facilitators } \\
\text { and barriers to adoption of epilepsy self-management } \\
\text { interventions }\end{array}$ & $\begin{array}{l}\text { - Self-described pilot studies and/or sample size }<20 \\
\text { - Studies with retrospective data collection } \\
\text { - Interrupted time series } \\
\text { - Case series } \\
\text { - Systematic reviews/meta-analyses } \\
\text { - Reports that do not include primary data on barriers or } \\
\text { facilitators }\end{array}$ \\
\hline Language & English & Non-English \\
\hline Countries & $\mathrm{OECD}^{c}$ & Non-OECD \\
\hline Years & Any & None \\
\hline $\begin{array}{l}\text { Publication } \\
\text { types }\end{array}$ & Full publication in a peer-reviewed journal & $\begin{array}{l}\text { Letters, editorials, reviews, dissertations, meeting abstracts, } \\
\text { protocols without results }\end{array}$ \\
\hline
\end{tabular}

${ }^{\text {a Adapted from Jonkman et al. [32] }}$

${ }^{b}$ See Cochrane EPOC criteria for definitions and details [33]

'OECD Organization for Economic Cooperation and Development includes Australia, Austria, Belgium, Canada, Chile, Czech Republic, Denmark, Estonia, Finland, France, Germany, Greece, Hungary, Iceland, Ireland, Israel, Italy, Japan, Korea, Latvia, Luxembourg, Mexico, Netherlands, New Zealand, Norway, Poland, Portugal, Slovak Republic, Slovenia, Spain, Sweden, Switzerland, Turkey, United Kingdom, United States

study design (e.g., qualitative, cross-sectional, mixedmethods) [35, 36] (Additional file 3). The evaluation of ROB in each study was based on an assessment of whether the study was well-designed and conducted within the research paradigm proposed by the study authors. These tools assess risk of bias in 5 to 10 domains, categorizing each domain as low, unclear, or high ROB. The tools are not intended to create summative scores $[35,36]$, and so we applied a more holistic approach by considering the individual domains and overall impressions of the study. First, each study was independently rated by each qualitative investigator (AAL, AS) using a ROB tool. Then, using this first ROB rating, the two investigators met and discussed each study in the context of valid and rigorous qualitative research methodology [37-39]. For instance, we discussed sampling technique (e.g., did the authors describe recruitment and sample selection in detail? Did the sampling align with the population described in the introduction?); analysis (e.g., Was analysis completed by one individual or more 
than one individual? Was analysis completed by the individual who completed data collection?); and measures (e.g., Does the data collection method align with the introduction and purpose of the study?). After discussing each study, we assigned an overall ROB using the following definitions: (1) low $R O B$-bias, if present, is unlikely to alter the results seriously; (2) unclear $R O B$-a risk of bias that raises some doubts about the results; and (3) high $R O B$ - bias may alter the results seriously. Disagreements were resolved by consensus between the two qualitative investigators and the senior author. We did not validate each ROB tool for use in our systematic review as we did not make any real change to the intent or the goal of each instrument.

We used the 10-item Critical Appraisal Skills Programme (CASP) for Qualitative Research Studies [35] for the included qualitative studies $(n=5)$. Each item is rated "Yes," "No," or "Can't tell"; there is no summary rating. The overall ROB in the 5 qualitative studies was low [22, 40-43]. For the quantitative descriptive studies, we used the 5 items in the Mixed Methods Appraisal Tool (MMAT) [36]. These criteria address the sampling strategy, the sample representativeness, measurements, risk of nonresponse bias, and appropriateness of the statistical analysis. The MMAT rates each item "Yes," "No," or "Can't tell"; there is no summary rating. We used the 5-item MMAT specific to mixed-methods studies [36] for the mixed-methods study $(n=1)$. These criteria address the rationale for using mixed-methods, the integration of the study components, the interpretation of the study components, discussion of divergences or inconsistencies between the quantitative and qualitative data, and how each component of the study adheres to the quality criteria of each of the quantitative and qualitative methods. Similar to the quantitative descriptive studies, the MMAT for mixed-methods studies rates each item "Yes," "No," or "Can't tell"; there is no summary rating.

\section{Data synthesis and presentation}

Data synthesis was completed by the same coinvestigators under the guidance of the primary investigator. We analyzed the abstracted data using thematic synthesis and the framework method $[44,45]$. Thematic synthesis utilizes a three-stage method for data synthesis: (1) free coding of findings in the included studies, (2) organizing data into descriptive themes, and (3) generating analytical themes that combine the findings of individual studies into interpretations that cross the findings of the studies [45]. The framework method is a complementary approach to management and analysis of qualitative data, which relies on charting the data into a matrix based on descriptive and analytic codes [44]. Using the research question and our full-text review and familiarization with these data as a guide, we created a framework based on three levels from the social ecological framework [46] that included barriers and facilitators as reported for a category (i.e., patient with epilepsy or caregiver, program or intervention, and site or health system). Then, we described each piece of data either as being a facilitator or a barrier to implementation of a self-management program. Each piece of data was subsequently grouped into an ecological framework level (i.e., patient/caregiver, program/intervention, site/ health system). We then completed first-level analysis of these data and confirmed the validity of our interpretations by referencing the original texts. After the data were independently coded and discussed among the two qualitative researchers, we conducted thematic synthesis by identifying and grouping related codes within each category (e.g., patient/caregiver, program/intervention, site/health system). Then, each researcher independently organized related codes into themes. We reviewed the themes and then identified overarching themes that applied to both facilitators and barriers. The creation and identification of codes and themes was iterative. To ensure rigor and validity of these findings, we independently coded and developed themes. We then discussed theme development and identification until we reached agreement between the two researchers. Next, we conducted a narrative synthesis of the data and displayed its summary in a crosstab of themes by study.

\section{Ecological levels}

For each facilitator and barrier, we first identified the respondent (e.g., the patient with epilepsy, caregiver, or health care clinician) associated with each theme. Then we examined each theme at one of three levels, adapted from ecological models of health behavior. The ecological models of health emphasize that determinants of behavior derive from individuals and characteristics of their environments that influence behavior directly and in interaction with one another [46]. Table 2 provides operationalized definitions and exemplars for each of the three ecological levels. These categories were mutually exclusive; thus, each facilitator and barrier could only be placed in one category (i.e., patient/caregiver, program/ intervention, site/health system).

\section{Results}

We identified 13 studies addressing facilitators and barriers to implementing self-management interventions for persons with epilepsy (Fig. 1) [22, 40-43, 47-54]. Study designs in this analysis included semi-structured interview $(n=5)$ [22, 40-43]; cross-sectional survey $(n=5)$ [47, 48, 50, 53, 54]; longitudinal survey $(n=1)$ [52], mixed-methods including group semi-structured interview, cross-sectional survey, and records review $(n=1)$ 
Table 2 Definitions and examples of the ecological levels

\begin{tabular}{lll}
\hline Level & Definition & Examples \\
\hline Person & $\begin{array}{l}\text { The individual characteristics of the patient or caregiver } \\
\text { who is engaging in the epilepsy self-management intervention }\end{array}$ & $\begin{array}{l}\text { - Individual's current engagement in self-management } \\
\text { behaviors [47] }\end{array}$ \\
Program & $\begin{array}{l}\text { Self-management intervention being implemented and } \\
\text { evaluated }\end{array}$ & $\begin{array}{l}\text { - Provision of relevant topics that enabled self-management } \\
\text { skill acquisition [42, 48] } \\
\text { - Involving caregivers in the intervention [49] }\end{array}$ \\
Site/system & $\begin{array}{l}\text { Health care site or system where the self-management } \\
\text { intervention is being implemented and evaluated that } \\
\text { highlights the usability of the intervention }\end{array}$ & $\begin{array}{l}\text { - Uniform program standards [42] } \\
\text { Provider characteristics such as job role within the } \\
\text { organization [50] }\end{array}$ \\
\hline
\end{tabular}

[49], and a discrete choice experiment $(n=1)$ [51]. Study respondents included patients with epilepsy, caregivers, and health care clinicians together [42, 49, 52$54]$, patients and caregivers only [22, 40, 41, 43, 47, 48, 51], and health care clinicians [50]. We describe study characteristics including study sample, epilepsy type, and intervention in Table 3.

Thematic synthesis identified 5 themes that could be applied conceptually to facilitators and barriers. These themes, described in Table 4, included relevance, personalization, intervention components, technology considerations, and clinician interventionist. The presence of themes varied by study as shown in Table 5 .

\section{Facilitators}

The presence of facilitators of epilepsy selfmanagement interventions at any level (i.e., person, program, site/system) was noted in 11 studies [22, 40-43, 47-50, 52, 54]. Two studies did not include any facilitators $[51,53]$.

\section{Relevance}

At the person level, facilitators included desire of the patient with epilepsy to acquire self-management skills [40, $54]$ and the desire to have content that was highly applicable to living with epilepsy (e.g., through eliciting concerns about self-managing and daily living from the

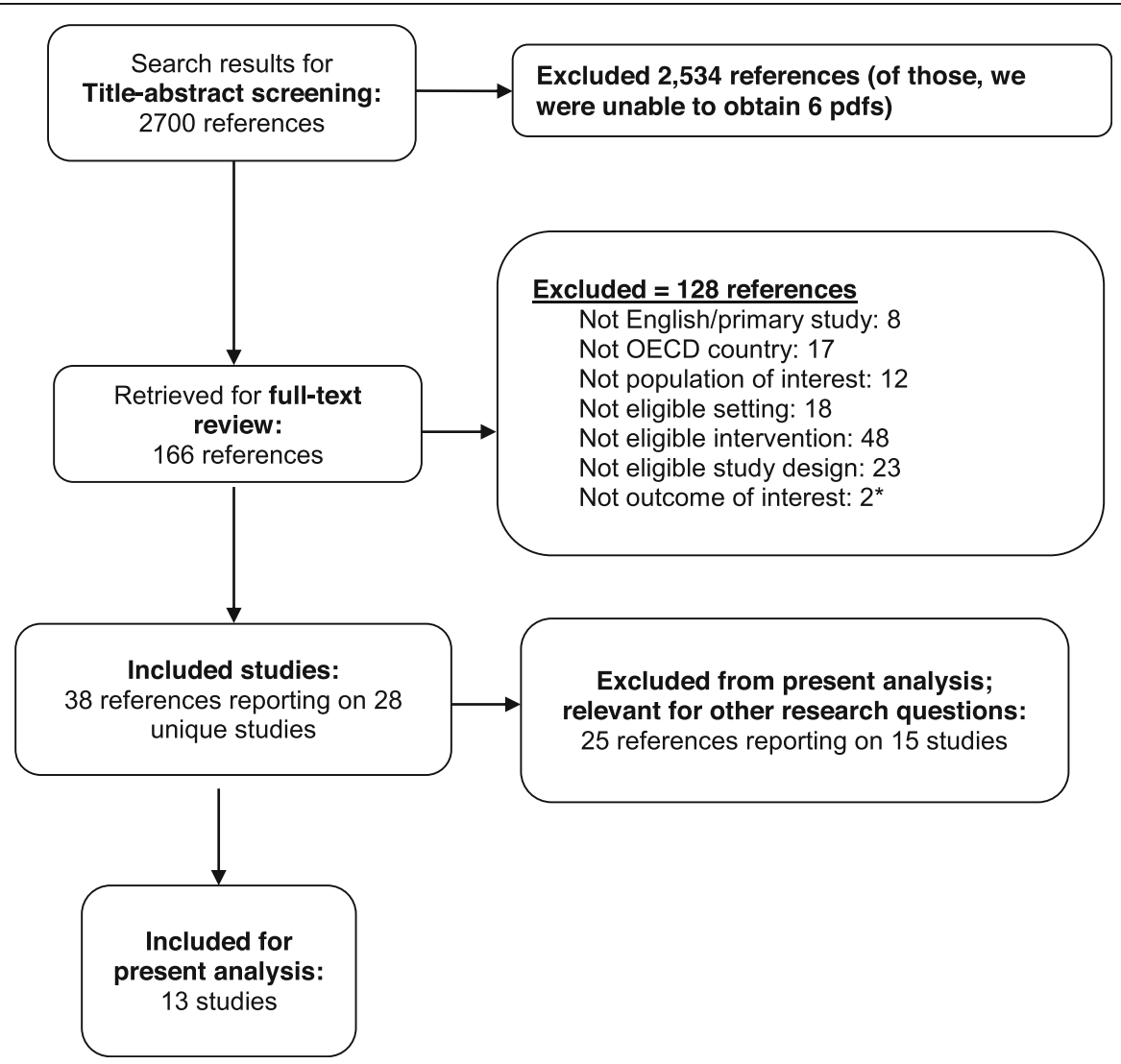

Fig. 1 PRISMA literature. OECD, Organization for Economic Cooperation and Development. Asterisk means reference did not report a relevant clinical, process, or economic outcome 


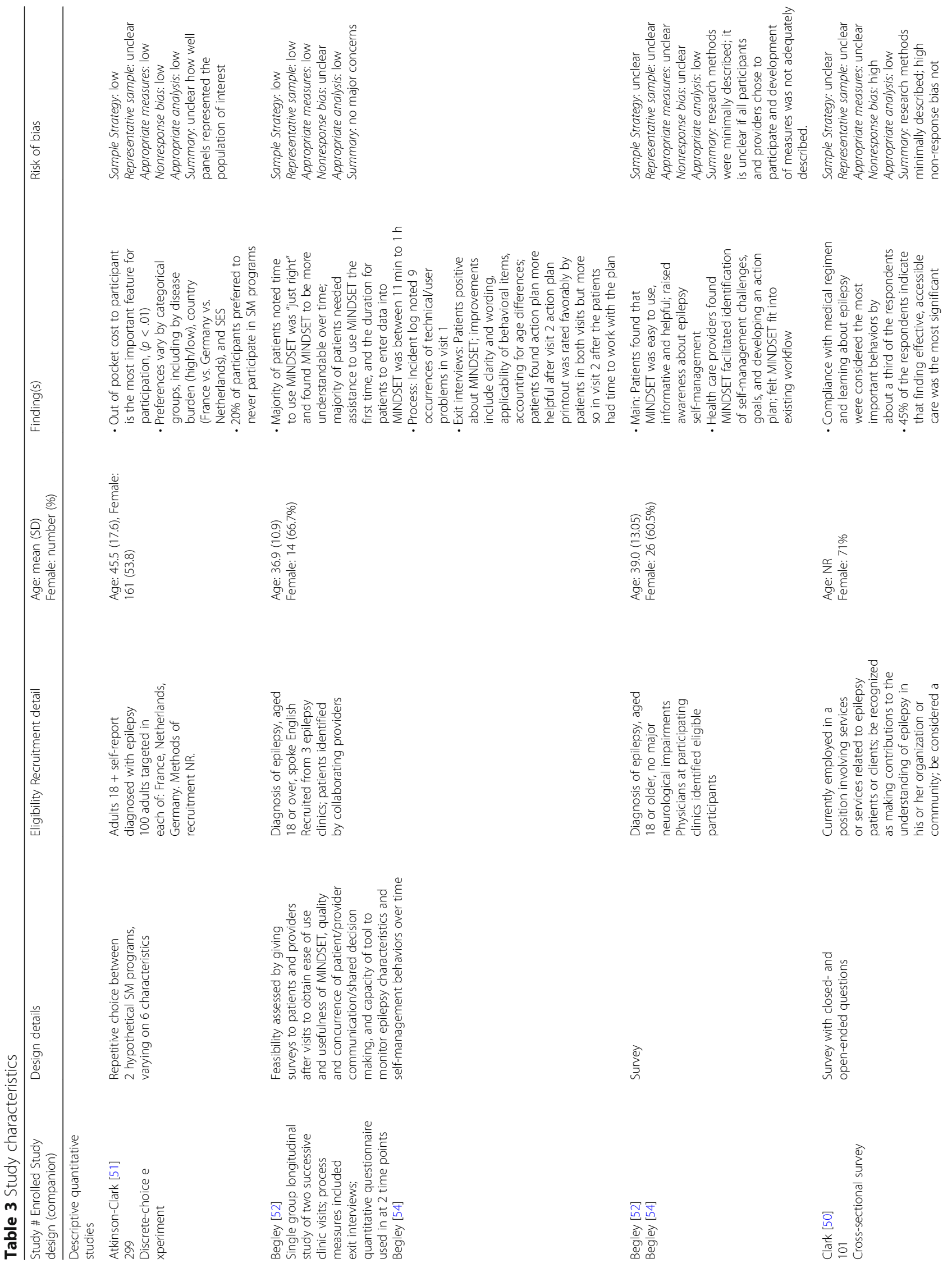




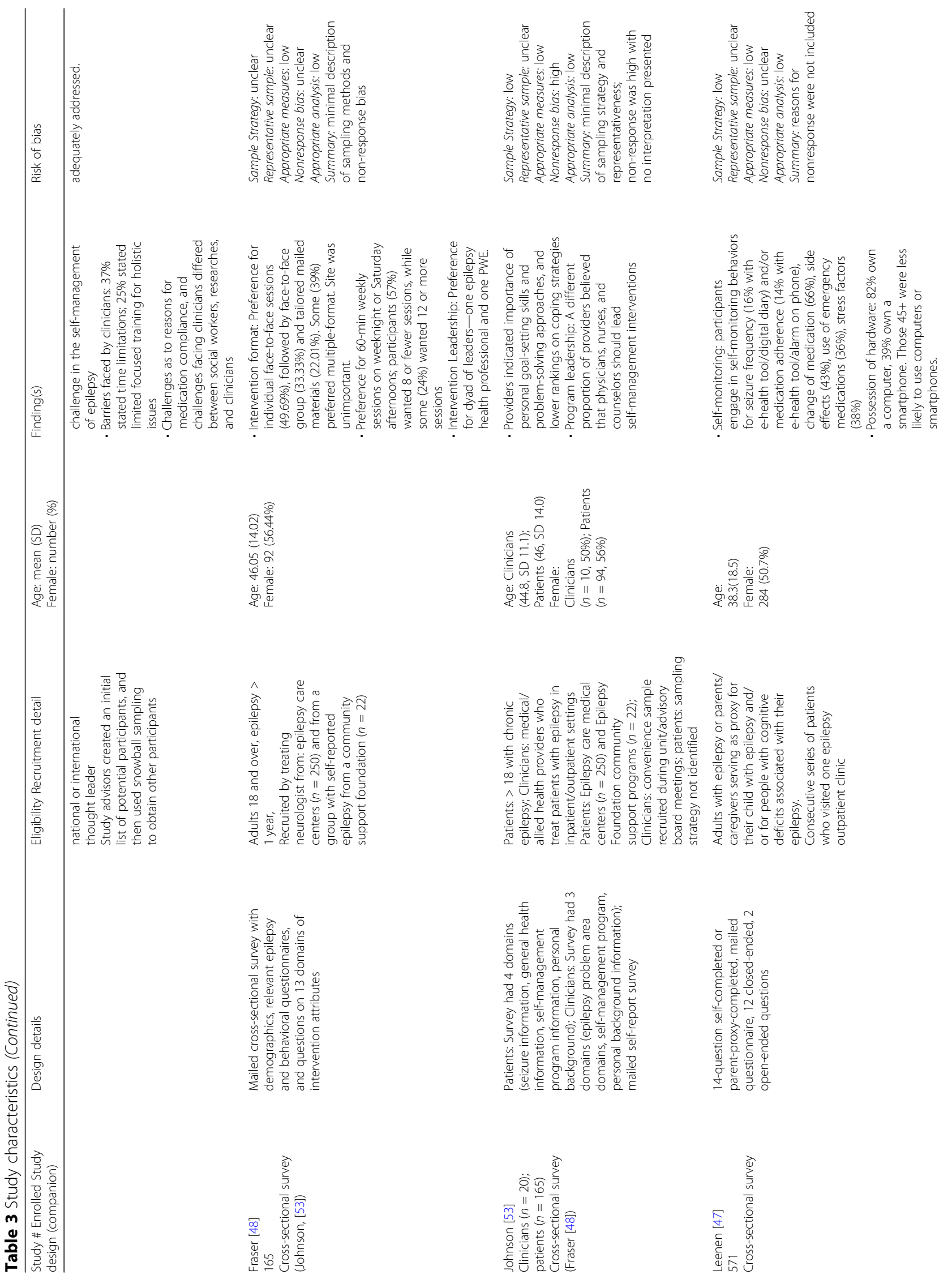




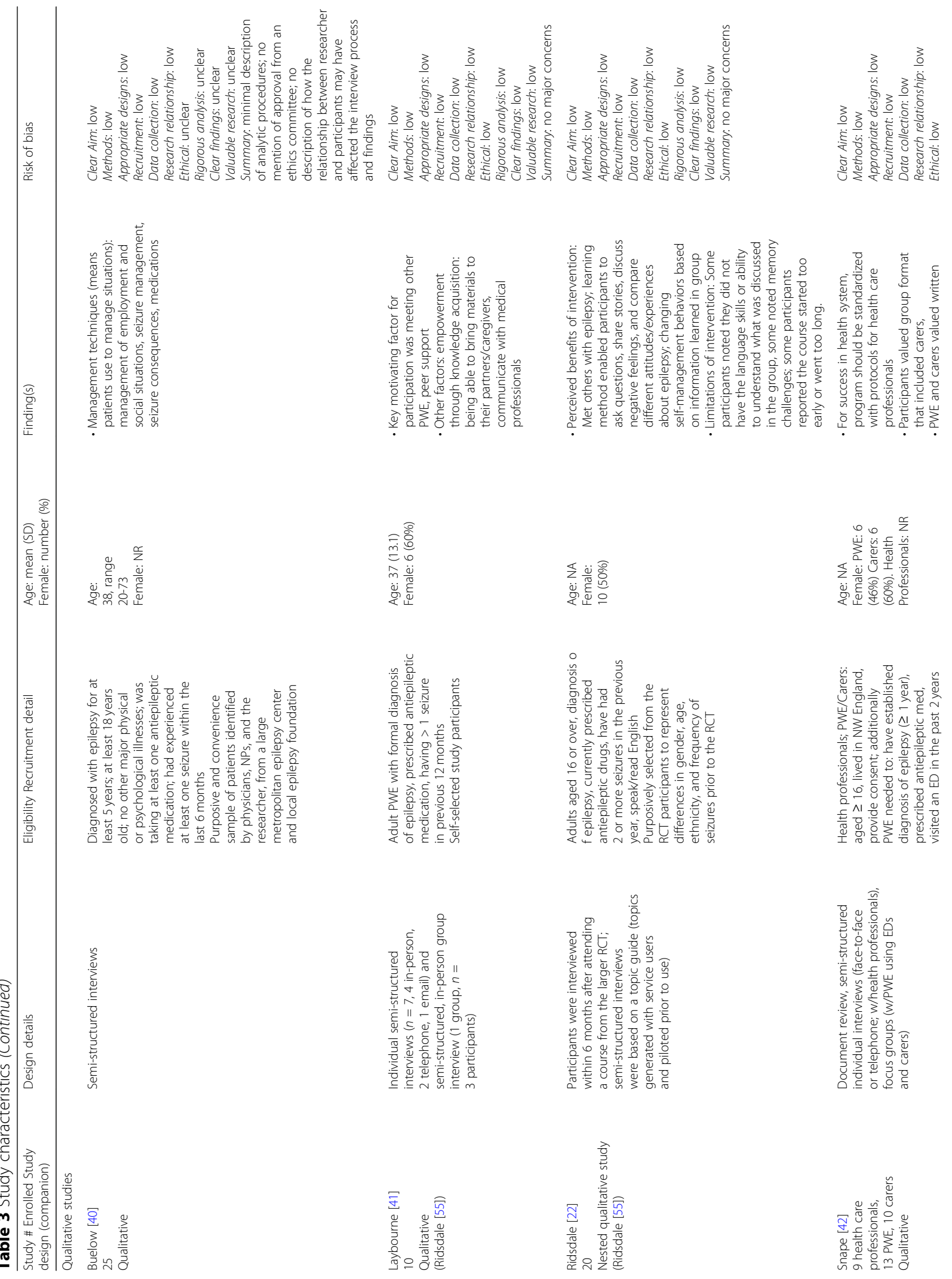


Lewinski et al. Systematic Reviews

(2020) 9:92

Page 10 of 17

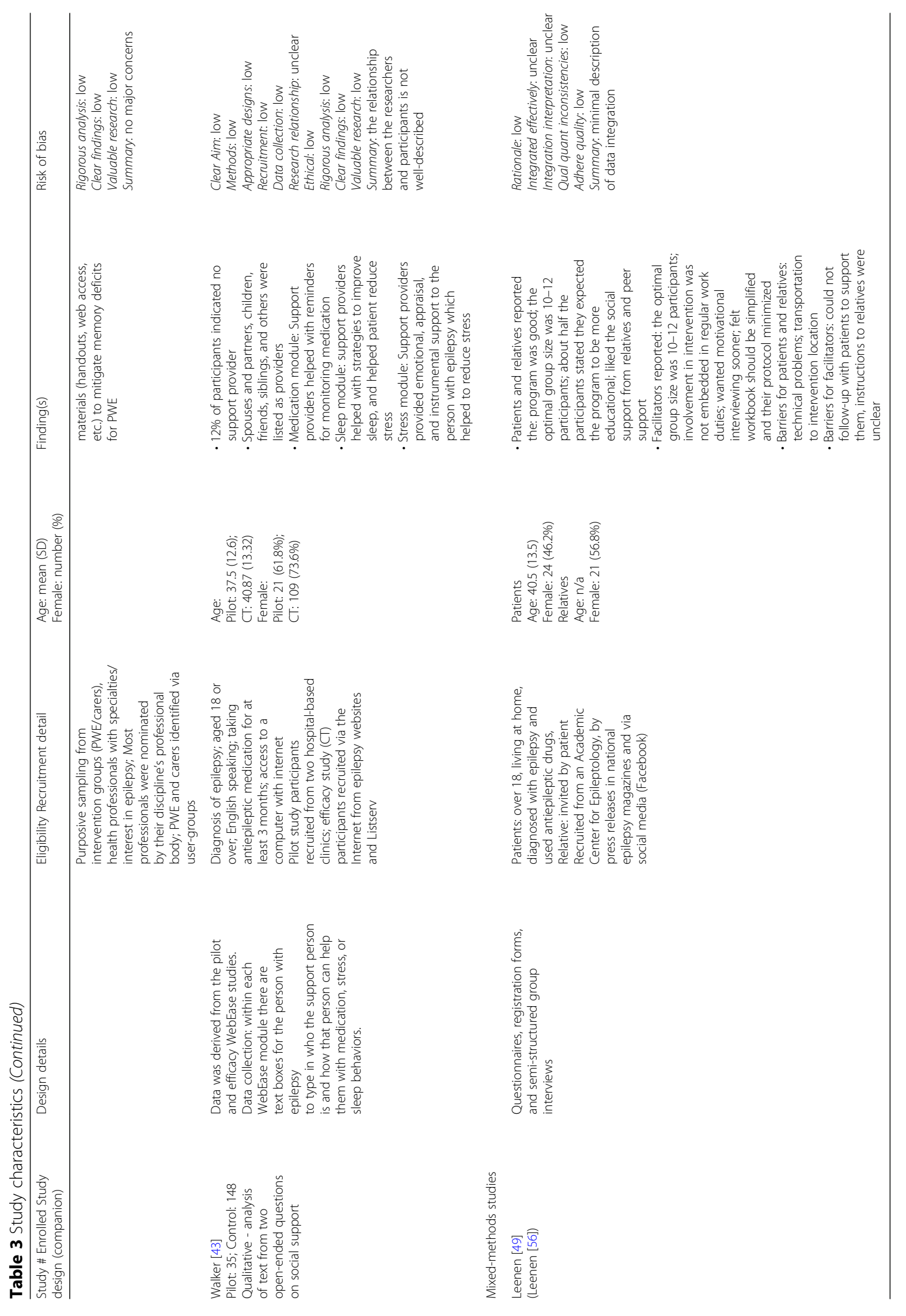


Table 4 Themes across studies of self-management of epilepsy

\begin{tabular}{ll}
\hline Theme & Definition \\
\hline Relevance & $\begin{array}{l}\text { Relevance of intervention content or topics } \\
\text { that facilitate the acquisition of self-management } \\
\text { skills in patients with epilepsy } \\
\text { Intervention components that account for } \\
\text { the individual social, physical, and } \\
\text { environmental characteristics of the patient } \\
\text { Components and dosing of the intervention } \\
\text { Intervention }\end{array} \quad \begin{array}{l}\text { Components } \\
\text { considerations }\end{array}$ \\
$\begin{array}{l}\text { Conse, familiarity with, and ownership of } \\
\text { technology (e.g., computers, laptops, } \\
\text { mobile phones) }\end{array}$ \\
interventionist
\end{tabular}

patient or caregiver) $(41,52,54)$. At the program level, facilitators included content designed to: enable the patient's acquisition of skills for living with epilepsy, learn how to apply self-management skills, develop coping strategies for daily life, and communicate with family, caregivers, and health care clinicians about epilepsy [41, $42,48,54]$. No relevance facilitators were identified at the site/system level.

\section{Personalization}

At the person level, facilitators included identifying whether the patient owned the necessary technology for the intervention (e.g., computer, mobile telephone) [47], had an identified source of social support (e.g., parents, significant others, friends) [43], and whether the intervention was congruent with the patient's preference for individual peer-support or group interaction $[41,42]$. At the program level, facilitators included developing the intervention and tailoring its components to build on the current self-management practices of the patient $[47,54]$. No personalization facilitators were identified at the site/system level.

\section{Intervention components}

At the person level, facilitators included patient or caregiver receiving written materials (e.g., educational content) to refer to during and after the intervention [42]. At the program level, facilitators included involving family members in the intervention [49], using an empowerment approach to help the patient develop self-management skills [41, 54], and the format of the intervention (e.g., group format that included both the patient and caregiver) [42]. Additional program level factors include the ability to personalize materials to each patient $[42,48,54]$, the availability of written materials $[41,42,52]$, the ability to interact with a group [22, 49], the provision of peer support
$[22,49]$, and the length and duration of the intervention sessions [22, 49]. At the site/system level, facilitators included developing intervention materials using uniform program standards to ensure program fidelity across intervention sites [42]. One study indicated that the site of the intervention (e.g., medical center) was unimportant, as patients with epilepsy indicated no preference of one site over another [48].

\section{Technology considerations}

At the program level, facilitators included the high level of usability of intervention components located on the internet, mobile applications, or phones $[47,52,54]$. No technology facilitators were identified at the person or site/system levels.

\section{Clinician interventionist}

At the program level, facilitators included creating an intervention team consisting of a patient in tandem with an expert health care clinician who could deliver the intervention content [48]. No clinician interventionist facilitators were identified at the person or site/system levels.

\section{Barriers}

The presence of barriers to epilepsy self-management interventions at any level was noted in 11 studies [22, 4143, 47, 49-54]. Two studies did not include any relevant barriers $[40,48]$. We noted that in the studies that addressed barriers, stakeholders included clinicians, social service providers, and researchers [50], and patients and clinicians [53].

\section{Relevance}

At the program level, barriers included incongruent responses between patients and clinicians about the patient's problems and needs [53], incongruent opinions by clinicians, researchers, and social service providers on the necessary intervention content [50], and incongruent responses between patients and clinicians on who should lead the intervention and provide epilepsy selfmanagement education and support [53]. Additional barriers at the program level included educational content that was too general or lacking in personalization or tailoring to the patient, his or her disease state and relevant comorbidities $[22,53,54]$, and not identifying what the patient views as important in self-management and living with epilepsy [49]. No relevance barriers were identified at the person or site/system levels.

\section{Personalization}

At the person level, barriers included the patient's memory and/or cognitive impairments [22, 42, 47, 50, 51, 53], the patient's disinterest in participating in a self- 
Lewinski et al. Systematic Reviews

(2020) 9:92

Page 12 of 17

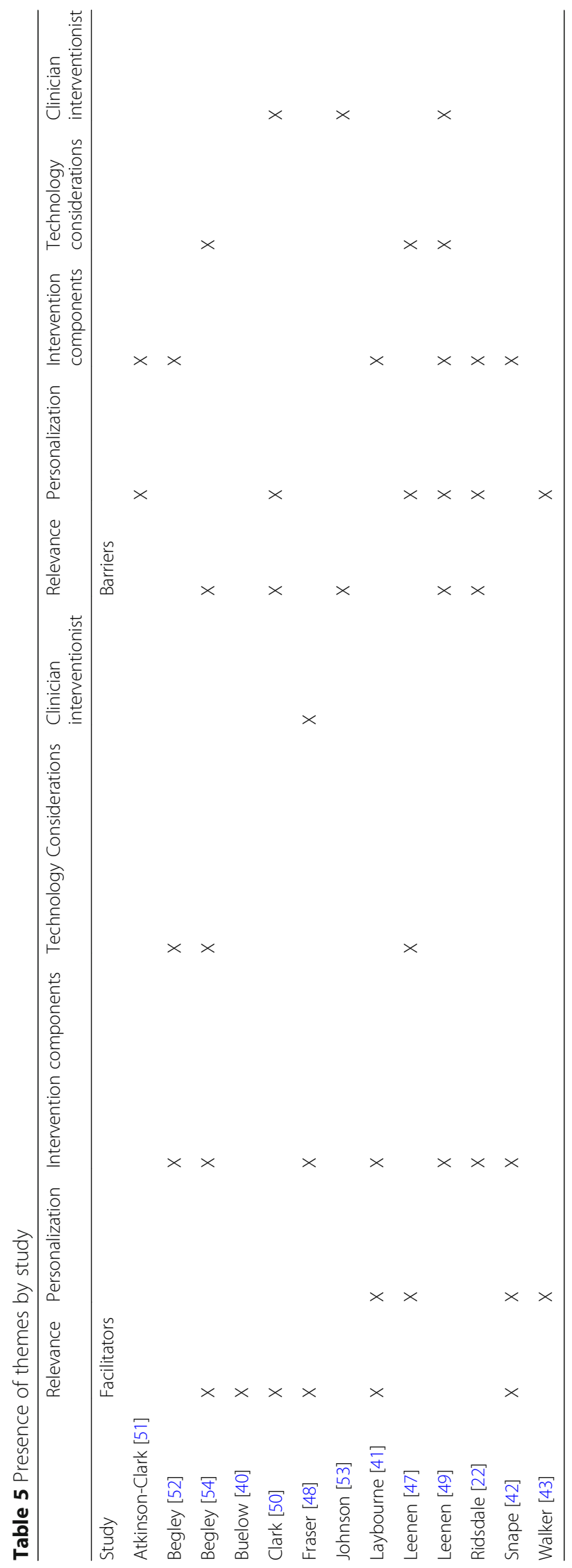


management intervention [51], not identifying the patient's preference or desire for self-management support [43], and no current use of the technology by the patient [47]. At the program level, barriers included not accounting for the cognitive limitations of the patient [22]. At the site/system level, barriers included not accounting for the characteristics of the patient population such as the patients' access to health care [50] or transportation concerns [49].

\section{Intervention components}

At the program level, barriers included having the patient incur a cost to participate in the intervention [51], not identifying how demographics (e.g., country of origin, burden of disease, socioeconomic status, level of activation) influence the patient's participation and views of the intervention [51], not identifying the ideal time for follow-up by the clinician after the intervention [49], and not having clear instructions for the role of caregivers participating in or affected by the intervention [49]. Additional program level barriers include not having written materials (e.g., program manuals, handouts, website resources) the patient can refer to during and after the intervention [41, 49], having groups heterogeneously composed of individuals with disparate experiences of living with epilepsy [22], experiencing challenges to scheduling group intervention sessions because of calendar conflicts for participants and clinicians [42], and not identifying the optimal duration and length of the intervention for patients [22, 41, 51]. Barriers at the site/system level included having different levels of attrition at study sites [52] and challenges to using a participatory approach to intervention development and content identification (e.g., lengthy time to complete, need to obtain ethical approval, and efforts to ensure participant engagement) [42]. No intervention component barriers were identified at the person level.

\section{Technology considerations}

At the person level, barriers included the patient's lack of knowledge about eHealth tools and functions, having concerns about the privacy of eHealth tools, and varying individual preferences for using technology for epilepsy selfmanagement [47]. At the program level, barriers included difficulty developing eHealth tools with high usability, and a lack of help for users encountering technical difficulties $[47,49,54]$. At the site/system level, barriers included not acknowledging or addressing the inequity of access to eHealth tools within the sample or the person with epilepsy's concerns about the privacy of eHealth tools [47].

\section{Clinician interventionist}

At the program level, barriers included not incorporating the duties of the intervention into the clinician interventionist's normal job duties (i.e., a collateral duty) [49], not adequately preparing the clinician interventionist to deliver the intervention content [49], developing a complex intervention protocol that is difficult to deliver [49], and not identifying the optimal professional role and educational training of the clinician interventionist $[50,53]$. At the site/system level, barriers included a lack of opportunity for the clinician interventionist to engage in continuity of care for the person with epilepsy following the conclusion of the intervention [49] and not accounting for the limited time allotted for medical visits $[50,54]$. No clinician interventionist barriers were identified at the person level.

\section{Risk of bias}

The tools used to assess risk of bias (ROB) (Table 3, Additional file 3) for the descriptive quantitative, mixedmethods, and qualitative studies did not provide for the calculation of summary scores for individual papers. Among the 7 descriptive quantitative studies, [47, 48, 50-54] ROB was heterogeneous. Patterns that led to judgments of higher ROB included unclear representativeness of the sample $(n=6)[47,48,50,51,53,54]$ high $(n=2)[50,53]$ or unclear $(n=4)[48,51,53,54]$ ROB from non-response, unclear risk of bias in sampling strategy $(n=3)[48,50,54]$ and unclear appropriateness of measures $(n=2)[50,54]$.

\section{Discussion}

This study is the first known systematic review of facilitators and barriers to implementation and adoption of epilepsy self-management interventions. This review aimed to identify facilitators and barriers by examining evidence from diverse studies. We identified five themes: relevance of intervention content, personalization of intervention content and methods, intervention components, technology considerations, and clinician interventionist considerations. Within these themes, facilitators and barriers were identified at the person, program, and system-levels. Our findings add to the literature as recent systematic reviews on self-management interventions for adults with epilepsy $[11,57,58]$ have not focused on facilitators and barriers to intervention implementation and adoption (Table 6).

Our findings underscore a number of key diseasespecific considerations for the implementation of epilepsy self-management interventions. Self-management practices that align with an individual's needs, values, and preferences are considered an important component in delivering patient-centered care $[59,60]$. Data in the present review indicates that interventions should be flexible in order to accommodate a person with epilepsy's preferences about peer support, group interactions, existing self-management strategies, existing selfmanagement social support, and technology preferences. Intervention materials should include written and 
portable self-management information that is personalized and tailored to the unique situation of each person with epilepsy. Notably, self-management information should be available prior to, during, and after intervention sessions so that individuals with epilepsy, and their caregivers can review and reference self-management content when engaging in self-management behaviors.

Epilepsy providers should incorporate delivery of selfmanagement education and skills into their regular clinical practice to enhance successful delivery of relevant self-management programmatic content. Incorporating the intervention duties into the epilepsy provider's role and duties will help ensure successful implementation of the intervention in practice and that the interventionist

Table 6 Highest priority evidence gaps

\begin{tabular}{|c|c|}
\hline $\begin{array}{l}\text { PICOTS } \\
\text { Domain }\end{array}$ & Evidence Gap \\
\hline Population & $\begin{array}{l}\text { Research is needed with patients who are earlier in their } \\
\text { course of illness and studies specifically focused on older } \\
\text { adults with epilepsy. Evaluation of interventions and } \\
\text { barriers/facilitators to implementation and adoption of } \\
\text { self-management interventions with patients and in large } \\
\text { health systems is missing. }\end{array}$ \\
\hline Interventions & $\begin{array}{l}\text { - Self-management interventions are needed that } \\
\text { incorporate patient, caregiver, and clinician interventionist } \\
\text { input, account for cognitive limitations, incorporate peer } \\
\text { support and address other barriers to engagement and } \\
\text { adherence. } \\
\text { - The role of technology (e.g., smart-phones, web-based } \\
\text { support) has not been well studied in patients with } \\
\text { epilepsy. } \\
\text { - Patients with epilepsy expressed a desire for an } \\
\text { intervention team composed of a person with epilepsy } \\
\text { and a clinician interventionist to provide self-management } \\
\text { education and support. Future research should further } \\
\text { examine the composition of this interventionist dyad and } \\
\text { identify who the clinician interventionist should be (e.g., } \\
\text { registered nurse, advanced practice registered nurse, } \\
\text { physician, physician assistant). } \\
\text { - Future research should focus on the extent to which } \\
\text { these intervention components (e.g., peer support), use } \\
\text { of technology, and other identified barriers/facilitators, } \\
\text { influence the person with epilepsy's initial and } \\
\text { sustained engagement in an epilepsy self-management } \\
\text { program. }\end{array}$ \\
\hline Comparators & $\begin{array}{l}\text { Active controls, including usual care, are appropriate and } \\
\text { should be described carefully. }\end{array}$ \\
\hline Outcomes & $\begin{array}{l}\text { Future research is needed that specifically addresses the } \\
\text { implementation and adoption of epilepsy } \\
\text { self-management programs, as there may be additional } \\
\text { personal, program, and site/system level barriers that } \\
\text { need to be identified and addressed. }\end{array}$ \\
\hline Timing & $\begin{array}{l}\text { Self-management skills can take time to master and may } \\
\text { take longer for patients with cognitive difficulty. } \\
\text { Consensus, or research, on the time required to acquire } \\
\text { self-management skills and the time required for new } \\
\text { skills to potentially improve clinical outcomes is needed. }\end{array}$ \\
\hline Setting & $\begin{array}{l}\text { Few studies have examined interventions delivered } \\
\text { outside of clinical settings. Future research should } \\
\text { determine the preferred location for a self-management } \\
\text { program for patients with epilepsy and their caregivers. }\end{array}$ \\
\hline
\end{tabular}

is appropriately trained in the intervention protocol. Overall, our findings indicate that the number of clinical personnel and the time requirements for participation in the intervention are important factors to consider when implementing and adopting interventions. However, no studies in our review addressed the costs related to implementing and adopting self-management programs for epilepsy, nor did any studies address clinician-related costs. Given the personnel and time required for epilepsy self-management programs, new models of care delivery (e.g., telemedicine, mHealth) should be considered as a means to facilitate implementing and adopting epilepsy self-management programs in a cost-effective manner. Virtual care delivery models may be less costly than face-to-face programs and may be more scalable, thus increasing the reach and impact of selfmanagement programs for persons with epilepsy.

One key finding of our study is that no studies directly addressed implementation and adoption issues for epilepsy self-management programs in large health systems. Our included studies addressed factors primarily at the patient level or program level. Important themes that could inform the development, implementation, and/or adoption of future epilepsy self-management interventions included the desire of patients with epilepsy to be involved in the development of intervention content and recognition that cognitive limitations may affect engagement and adherence. Stakeholder engagement is particularly important in developing self-management programs for epilepsy as differences exist among individuals with epilepsy and clinicians in regard to reasons for nonadherence and factors that impact epilepsy selfmanagement [30, 61, 62]. Involving key stakeholders (e.g., patients, caregivers, providers, administrators) may help promote facilitators, and attenuate barriers, to implementing and adopting an epilepsy selfmanagement program. Of note, no studies addressed facilitators and barriers to implementing and adopting epilepsy self-management interventions in relation to costeffectiveness. Therefore, future research should focus on identifying cost-effective strategies that promote facilitators and reduce barriers to implementation and adoption of epilepsy self-management programs.

Persons with epilepsy expressed a desire for a team composed of an individual with epilepsy and a clinician interventionist to deliver self-management education and support. Thus, future research should further examine the composition of this dyad and identify who the interventionist should be (e.g., registered nurse, advanced practice registered nurse, physician, physician assistant, etc.). One study identified that persons with epilepsy did not have a preference to intervention site such as a medical center or other location. Future research should determine the 
preferred location for a self-management program for persons with epilepsy and caregivers of persons with epilepsy, with an awareness that access to public transportation may vary widely within a community. Across the studies, persons with epilepsy stated several factors such as access to health care, familiarity and ownership of technology, finances, current selfmanagement support, and transportation that influenced intervention engagement. Future research should focus on the extent to which these factors either individually or in combination influence the person with epilepsy's initial and sustained engagement in an epilepsy self-management program. We did not identify any study that focused explicitly on the facilitators and barriers to the implementation and adoption of self-management programs for persons with epilepsy for a large health system. Future research is needed that specifically addresses the implementation and adoption of epilepsy self-management programs, as there may be additional personal, program, and site/system level barriers that need to be identified and addressed.

\section{Limitations and strengths}

This is one of the first known systematic reviews to apply rigorous qualitative methods to synthesize data from studies with a wide range of designs (e.g., crosssectional and longitudinal surveys, discrete-choice experiments, semi-structured interviews, records review, and mixed-methods designs). Using these qualitative evidence synthesis methods, we were able to obtain a more complete understanding of the phenomenon of facilitators and barriers to implementation of epilepsy self-management programs than by comparative methods alone. Yet, several limitations of our study need to be acknowledged. First, while we made every attempt to identify and locate interventions and manuscripts that report on the implementation of epilepsy self-management programs, we may have inadvertently missed relevant articles. Additionally, we limited our search to Organization for Economic Cooperation and Development countries; this search limitation may have inadvertently excluded relevant self-management interventions completed in other countries that could have provided valuable insight into the implementation of epilepsy self-management programs in large health systems. Second, the tools used to assess the risk of bias (ROB) for the studies included in the present review did not allow for the calculation of summary scores. We assessed all studies for ROB. Of the 13 studies, only 1 exhibited a high ROB due to insufficient information about ethical concerns, lack of rigorous analysis of study findings, and no clear value of the research. The remaining studies exhibited either low or unclear ROB. Additionally, we did not contact authors of the studies included in this review to clarify questions pertaining to methods or data collection. Contacting authors to obtain additional information on methods may have influenced our ROB assessment. For the studies in the present review, we addressed the inherent diversity by identifying the respondent (e.g., patient with epilepsy, caregiver, or clinician) and then synthesizing emerging themes within ecological levels.

Our study has a number of strengths. Our review benefited from leveraging input from content experts, being protocol driven, and using rigorous qualitative methods for analyzing barriers and facilitators to implementation and adoption. The use of a theorybased, a priori framework, and a thorough, multipleinvestigator review process ensured rigor and validity of our process, findings, and interpretation. By not limiting our inclusion criteria to either quantitative or qualitative designs, we were able to obtain a more comprehensive picture of relevant facilitators and barriers to implementation and adoption of epilepsy selfmanagement programs.

\section{Conclusion}

Findings on facilitators and barriers to implementation underscore key considerations for the design, implementation, and adoption of self-management interventions, including factors of patient personalization, information delivery, use of technology, and intervention personnel. Future research should be designed to address these implementation issues. Future research also should focus on the extent to which intervention components (e.g., peer support), use of technology, and other identified barriers/facilitators influence the person with epilepsy's initial and sustained engagement in an epilepsy selfmanagement program. In conclusion, our data indicate the importance of incorporating disease-specific considerations when designing, implementing, and adopting an epilepsy self-management program.

\section{Supplementary information}

Supplementary information accompanies this paper at https://doi.org/10. 1186/s13643-020-01322-9.

Additional file 1. PRISMA 2019 Checklist

Additional file 2. Search Strategy

Additional file 3. Risk of Bias (ROB) Assessment

\section{Abbreviations}

OECD: Organization for Economic Cooperation and Development; ROB: Risk of bias

\section{Acknowledgements}

The authors thank Glenn Graham, MD, PhD, for nominating the topic and providing input on project scope and methodology; Jennifer Patterson, PhD, 
and Paul Rutecki, MD, for input on project scope and methodological approaches; and Liz Wing, MA, for editorial assistance.

\section{Consent for publications}

Not applicable

\section{Declarations}

The findings and conclusions in this document are those of the author(s) who are responsible for its contents and do not represent the views of the Department of Veterans Affairs, the US Government, the Department of Defense or its Components, or Duke University. Therefore, no statement in this article should be construed as an official position of the Department of Veterans Affairs, the Department of Defense or its Components, or Duke University.

\section{Authors' contributions}

$A A L$ and AS screened abstracts, read full-text manuscripts, abstracted and analyzed data from relevant manuscripts, and wrote the manuscript. JMG, KG, and AMG, screened abstracts, read full-text manuscripts, assisted with data interpretation, and manuscript editing. JWW led the conduct of the project, screened abstracts, read full-text manuscripts, assisted with data interpretation, and manuscript writing. DVB, MWL, HBB, CD, JDL, SRS, TT, and AMH screened abstracts, assisted with data interpretation, and manuscript editing. MGV served as the medical librarian and assisted in obtaining relevant literature. The authors read and approved the final manuscript.

\section{Funding}

This work was supported by the Center of Innovation for Health Services Research in Primary Care (CIN 13-410) at the Durham VA and the VA Evidence-based Synthesis Program (ESP 09-010). AAL and DVB were supported by a VA OAA HSR\&D PhD Fellowship TPH 21-000. KG was supported by VA HSR\&D (CDA\# 13-263). HBB was supported by a Research Career Scientist award from VA HSR\&D (VA HSR\&D 08-027)

\section{Availability of data and materials}

Study protocol: Available at www.crd.york.ac.uk/prospero (PROSPERO: CRD42018098604). Qualitative data set: Available from Ms. Gordon (e-mail, adelaide.gordon@va.gov).

\section{Ethics approval and consent to participate}

Not applicable

\section{Competing interests}

Dr. Luedke reports grants, personal fees, and other (participation for charitable work) from Eisai Pharmaceuticals and grants from Biogen and UCB, outside the submitted work. Dr. Sinha reports grants and personal fees from UCB; personal fees from Monteris, Cadwell, and Springer Clinical Medicine; and nonfinancial support from Sunovion, the American Clinical Neurophysiology Society, the American Board of Clinical Neurophysiology, and ABRET Neurodiagnostic Credentialing and Accreditation, outside the submitted work. Dr. Husain reports personal fees and nonfinancial support from Jazz Pharmaceuticals; personal fees from Biogen Idec, Eisai, and Neurelis; and grants and personal fees from Marinus Pharmaceuticals, outside the submitted work; royalties from Springer Publishing, Demos Medical Publishing, and Wolters Kluwer; and an editorship stipend from the American Clinical Neurophysiology Society. Dr. Bosworth reports grants and personal fees from Sanofi and Otuska; grants from Improved Patient Outcomes, Novo Nordisk, Novartis, Proteus, the US Department of Veterans Affairs, and the National Institutes of Health; and personal fees from Abbott, outside the submitted work. Dr. Williams reports grants from the VHA during the conduct of the study. The remaining authors report no competing interests.

\section{Author details}

'Durham Center of Innovation to Accelerate Discovery and Practice Transformation, Durham Veterans Affairs Medical Center, Durham, NC, USA ${ }^{2}$ Cooperative Studies Program Epidemiology Center-Durham, Durham Veterans Affairs Medical Center, Durham, NC, USA. ${ }^{3}$ Department of Population Health Sciences, Duke University School of Medicine, Durham, NC, USA. ${ }^{4}$ Division of General Internal Medicine, Department of Medicine, Duke University School of Medicine, Durham, NC, USA. ${ }^{5}$ Department of
Psychiatry and Behavioral Sciences, Duke University School of Medicine Durham, NC, USA. ${ }^{6}$ Department of Neurology, Duke University Medical Center, Durham, NC, USA. ${ }^{7}$ Neurodiagonostic Center, Durham Veterans Affairs Medical Center, Durham, NC, USA. ${ }^{8}$ School of Nursing, Duke University, Durham, NC, USA. ${ }^{9}$ Department of Health Policy and Management, Gillings School of Global Public Health, University of North Carolina at Chapel Hill, Chapel Hill, NC, USA. ${ }^{10}$ Center for Personalized Health Care, Duke University School of Medicine, Durham, NC, USA. ${ }^{11}$ Department of Neurology, Uniformed Services University School of Medicine, Bethesda, MD, USA. ${ }^{12}$ Neuroscience Medicine, Duke Clinical Research Institute, Duke University, Durham, NC, USA. ${ }^{13}$ University of California at Davis, Davis, CA, USA.

Received: 31 October 2019 Accepted: 6 March 2020

Published online: 25 April 2020

\section{References}

1. World Health Organization. Improving access to epilepsy care. Available at: https://www.who.int/mental_health/neurology/epilepsy/en/. Accessed 20 Dec 2018.

2. Kanner AM. Management of psychiatric and neurological comorbidities in epilepsy. Nat Rev Neurol. 2016;12(2):106-16.

3. Nevalainen $\mathrm{O}$, Ansakorpi $\mathrm{H}$, Simola M, et al. Epilepsy-related clinical characteristics and mortality: a systematic review and meta-analysis. Neurology. 2014;83(21):1968-77.

4. Thurman DJ, Logroscino G, Beghi E, et al. The burden of premature mortality of epilepsy in high-income countries: a systematic review from the Mortality Task Force of the International League Against Epilepsy. Epilepsia. 2017:58(1):17-26.

5. Samsonsen C, Reimers A, Brathen G, Helde G, Brodtkorb E. Nonadherence to treatment causing acute hospitalizations in people with epilepsy: an observational, prospective study. Epilepsia. 2014;55(11):e125-8.

6. Devinsky $O$, Ryvlin P, Friedman D. Preventing sudden unexpected death in epilepsy. JAMA Neurol. 2018;75(5):531-2.

7. Helgeson DC, Mittan R, Tan SY, Chayasirisobhon S. Sepulveda Epilepsy education: the efficacy of a psychoeducational treatment program in treating medical and psychosocial aspects of epilepsy. Epilepsia. 1990;31(1): $75-82$.

8. May TW, Pfafflin M. The efficacy of an educational treatment program for patients with epilepsy (MOSES): results of a controlled, randomized study. Modular Service Package Epilepsy. Epilepsia. 2002;43(5):539-49.

9. Luedke MW, Blalock DV, Goldstein KM, et al. Self-management of epilepsy: a systematic review. Ann Intern Med. 2019.

10. Luedke MW, Blalock DV, Lewinski AA, et al. Self-management of epilepsy. VA ESP Project \#09-009. 2018.

11. Bradley PM, Lindsay B, Fleeman N. Care delivery and self management strategies for adults with epilepsy. The Cochrane database of systematic reviews. 2016:2:CD006244.

12. Liporace J, D'Abreu A. Epilepsy and women's health: family planning, bone health, menopause, and menstrual-related seizures. Mayo Clin Proc. 2003; 78(4):497-506

13. Smaldone M, Sukkarieh T, Reda A, Khan A. Epilepsy and erectile dysfunction: a review. Seizure. 2004;13(7):453-9.

14. Morrell MJ. Stigma and epilepsy. Epilepsy Behav. 2002;3(6S2):21-5.

15. Tian N, Boring M, Kobau R, Zack MM, Croft JB. Active epilepsy and seizure control in adults - United States, 2013 and 2015. MMWR Morb Mortal Wkly Rep. 2018;67(15):437-42

16. Buck D, Baker GA, Chadwick DW, Jacoby A. Factors influencing compliance with antiepileptic drug regimes. Seizure. 1997;6(2):87-93.

17. Faroog S, Naeem F. Tackling nonadherence in psychiatric disorders: current opinion. Neuropsychiatr Dis Treat. 2014;10:1069-77.

18. U.S. Centers For Disease Controls and Prevention Epilepsy Program. About one-half of adults with active epilepsy and seizures have annual family incomes under \$25,000: The 2010 and 2013 US National Health Interview Surveys. Epilepsy Behav. 2016;58:33-4.

19. Bautista RED. Understanding the self-management skills of persons with epilepsy. Epilepsy Behav. 2017;69:7-11.

20. Dilorio C, Osborne Shafer P, Letz R, Henry T, Schomer DL, Yeager K. The association of stigma with self-management and perceptions of health care among adults with epilepsy. Epilepsy \& behavior : E\&B. 2003;4(3):259-67. 
21. Simblett SK, Bruno E, Siddi S, et al. Patient perspectives on the acceptability of mHealth technology for remote measurement and management of epilepsy: a qualitative analysis. Epilepsy \& behavior : E\&B. 2019;97:123-9.

22. Ridsdale L, Philpott SJ, Krooupa AM, Morgan M. People with epilepsy obtain added value from education in groups: results of a qualitative study. Eur J Neurol. 2017;24(4):609-16.

23. Bower $\mathrm{P}$, Kennedy $\mathrm{A}$, Reeves $\mathrm{D}$, et al. A cluster randomised controlled trial of the clinical and cost-effectiveness of a 'whole systems' model of selfmanagement support for the management of long- term conditions in primary care: trial protocol. Implement Sci. 2012;7:7.

24. Davy C, Bleasel J, Liu H, Tchan M, Ponniah S, Brown A. Factors influencing the implementation of chronic care models: a systematic literature review. BMC Fam Pract. 2015;16(1):102.

25. Shaw RJ, Kaufman MA, Bosworth HB, et al. Organizational factors associated with readiness to implement and translate a primary care based telemedicine behavioral program to improve blood pressure control: the HTN-IMPROVE study. Implement Sci. 2013;8(1):106.

26. Kadu MK, Stolee P. Facilitators and barriers of implementing the chronic care model in primary care: a systematic review. BMC Fam Pract. 2015;16:12.

27. Perrin KM, Burke SG, O'Connor D, et al. Factors contributing to intervention fidelity in a multi-site chronic disease self-management program. Implement Sci. 2006;1:26.

28. Centers for Disease Control and Prevention. Managing epilepsy well network: putting collective wisdom to work for people with epilepsy. In: Atlanta, GA.: Centers for disease control and prevention (US), National Center for Chronic Disease Prevention and Health Promotion; 2013: https:// static1.squarespace.com/static/5b4cd1975417fcf45807b53c/t/5b55eeb86d2 a737f2cdc7e8f/1532358339227/MEW_Booklet_

PuttingCollectiveWisdomToWork.pdf. Accessed January 20th, 2020.

29. Institute of Medicine. Epilepsy across the spectrum: promoting health and understanding. Available at: https://www.nap.edu/catalog/13379/epilepsyacross-the-spectrum-promoting-health-and-understanding. Accessed 22 Oct 2018.

30. Helmers SL, Kobau R, Sajatovic M, et al. Self-management in epilepsy: why and how you should incorporate self-management in your practice. Epilepsy Behav. 2017:68:220-4.

31. Moher D, Liberati A, Tetzlaff J, Altman DG. Preferred reporting items for systematic reviews and meta-analyses: the PRISMA statement. PLoS Med. 2009;6(7):e1000097.

32. Jonkman NH, Schuurmans MJ, Jaarsma T, Shortridge-Baggett LM, Hoes AW, Trappenburg JC. Self-management interventions: proposal and validation of a new operational definition. J Clin Epidemiol. 2016;80:34-42.

33. Cochrane Effective Practice and Organisation of Care (EPOC). Suggested risk of bias criteria for EPOC reviews. EPOC Resources for review authors, 2017. Available at: http://epoc.cochrane.org/resources/epoc-resources-reviewauthors Accessed 17 May 2018

34. Department of Veterans Health Administration HSRD, Quality enhancement research fnitiative,. Implementation Guide. In:2013.

35. Critical Appraisal Skills Programme (CASP). CASP qualitative checklist Available at: http://www.casp-uk.net/casp-tools-checklists. Accessed 17 May 2018.

36. Hong QN, Pluye P, Fàbregues S, et al. Mixed methods appraisal tool (MMAT), version 2018. Registration of Copyright (\#1148552), Canadian Intellectual Property Office, Industry Canada. Available at: http:// mixedmethodsappraisaltoolpublic.pbworks.com/w/page/127425845/ Download\%20the\%20MMAT. Accessed 11 Oct 2018.

37. Sandelowski M, Barroso J. Reading qualitative studies. Int J Qual Methods. 2002;1(1):74-108.

38. Devers KJ. How will we know "good" qualitative research when we see it? Beginning the dialogue in health services research. Health Serv Res. 1999; 34(5 Pt 2):1153-88.

39. Maxwell J. Understanding and validity in qualitative research. Harv Educ Rev. 1992;62(3):279-300.

40. Buelow JM. Epilepsy management issues and techniques. J Neurosci Nurs. 2001;33(5):260-9.

41. Laybourne AH, Morgan M, Watkins SH, Lawton R, Ridsdale L, Goldstein LH. Self-management for people with poorly controlled epilepsy: Participants'views of the UK Self-Management in eplLEpsy (SMILE) program. Epilepsy Behav. 2015;52(Pt A):159-164.

42. Snape DA, Morgan M, Ridsdale L, Goodacre S, Marson AG, Noble AJ. Developing and assessing the acceptability of an epilepsy first aid training intervention for patients who visit UK emergency departments: a multimethod study of patients and professionals. Epilepsy Behav. 2017;68:177-85.

43. Walker ER, Bamps Y, Burdett A, Rothkopf J, Diiorio C. Social support for selfmanagement behaviors among people with epilepsy: a content analysis of the WebEase program. Epilepsy Behav. 2012;23(3):285-90.

44. Gale NK, Heath G, Cameron E, Rashid S, Redwood S. Using the framework method for the analysis of qualitative data in multi-disciplinary health research. BMC Med Res Methodol. 2013;13:117.

45. Thomas J, Harden A. Methods for the thematic synthesis of qualitative research in systematic reviews. BMC Med Res Methodol. 2008:8:45.

46. Sallis JF, Owen N, Fisher E. Ecological models of health behavior. In: Health behavior: theory, research, and practice. 5th Edition. 2015:43-64.

47. Leenen LAM, Wijnen BFM, de Kinderen RJA, van Heugten CM, Evers SMAA, Majoie MHJM. Are people with epilepsy using eHealth-tools? Epilepsy Behav. 2016;64(Part A):268-272

48. Fraser RT, Johnson EK, Miller JW, et al. Managing epilepsy well: selfmanagement needs assessment. Epilepsy Behav. 2011;20(2):291-8.

49. Leenen LAM, Wijnen BFM, van Haastregt JCM, et al. Process evaluation of a multi-component self-management intervention for adults with epilepsy (ZMILE study). Epilepsy Behav. 2017;73:64-70.

50. Clark NM, Stoll S, Youatt EJ, Sweetman M, Derry R, Gorelick A. Fostering epilepsy self management: the perspectives of professionals. Epilepsy Behav. 2010;19(3):255-63.

51. Atkinson-Clark E, Charokopou M, Van Osselaer N, Hiligsmann M. A discretechoice experiment to elicit preferences of patients with epilepsy for selfmanagement programs. Epilepsy Behav. 2018;79:58-67.

52. Begley C, Shegog R, Harding A, Goldsmith C, Hope O, Newmark M. Longitudinal feasibility of MINDSET: a clinic decision aid for epilepsy selfmanagement. Epilepsy Behav. 2015;44:143-50.

53. Johnson EK, Fraser RT, Miller JW, et al. A comparison of epilepsy selfmanagement needs: provider and patient perspectives. Epilepsy Behav. 2012;25(2):150-5.

54. Begley C, Chong J, Shegog R, et al. MINDSET: Clinical feasibility of utilizing the revised epilepsy self-management tool for Spanish speaking patients. Epilepsy Behav. 2018;88:218-26.

55. Ridsdale L, McKinlay A, Wojewodka G et al. Self-Management education for adults with poorly controlled epILEpsy [SMILE (UK)]: a randomised controlled trial. Health Technol Assess. 2018;22(21):1-142.

56. Leenen LAM, Wijnen BFM, Kessels AGH et al. Effectiveness of a multicomponent self-management intervention for adults with epilepsy (ZMILE study): A randomized controlled trial. Epilepsy Behav. 2018;80: 259-65.

57. Smith A, McKinlay A, Wojewodka G, Ridsdale L. A systematic review and narrative synthesis of group self-management interventions for adults with epilepsy. BMC Neurol. 2017;17(1):114.

58. Fitzsimons M, Normand C, Varley J, Delanty N. Evidence-based models of care for people with epilepsy. Epilepsy Behav. 2012;23(1):1-6.

59. Lorig KR, Holman H. Self-management education: history, definition, outcomes, and mechanisms. Ann Behav Med. 2003;26(1):1-7.

60. U.S. Department of Veterans Affairs. Whole Health For Life. Available at: https://www.va.gov/patientcenteredcare/. Accessed 13 Dec 2018.

61. Sauro KM, Jette N, Quan H, Holroyd-Leduc J, DeCoster C, Wiebe S. Improving knowledge translation of clinical practice guidelines for epilepsy. Epilepsy Behav. 2019;92:265-8.

62. Groenewegen A, Tofighy A, Ryvlin P, Steinhoff BJ, Dedeken P. Measures for improving treatment outcomes for patients with epilepsy--results from a large multinational patient-physician survey. Epilepsy \& behavior : E\&B. 2014;34:58-67.

\section{Publisher's Note}

Springer Nature remains neutral with regard to jurisdictional claims in published maps and institutional affiliations. 